\title{
Transport of gadolinium in a cement composite
}

\author{
Igor Medved ${ }^{1,3,{ }^{*}, \text { Ladislav Kalvoda }}{ }^{2}$, Eva Vejmelková $^{1}$, Stanislav Vratislav ${ }^{2}$, and Robert \\ Cerný ${ }^{1}$ \\ ${ }^{1}$ Department of Materials Engineering and Chemistry, Faculty of Civil Engineering, Czech Technical \\ University, 16629 Prague, Czech Republic \\ ${ }^{2}$ Department of Solid State Engineering, Faculty of Nuclear Science and Physical Engineering, Czech \\ Technical University, 11519 Prague, Czech Republic \\ ${ }^{3}$ Department of Physics, Faculty of Civil Engineering, Slovak University of Technology, 81005 \\ Bratislava, Slovakia
}

\begin{abstract}
Development of cement based composites suitable for radionuclide immobilization is of great interest for secure containment of nuclear waste. This requires, in particular, detailed knowledge of how radionuclides are transported in such composites. In this paper we investigate the transport of gadolinium in a dry cement composite. Experimental data are obtained in a non-destructive way by neutron imaging. Their theoretical interpretation is based on a diffusion-advection model in which sorption is included.
\end{abstract}

\section{Introduction}

The assessment of whether a nuclear waste repository will meet necessary safety standards for long periods of time $\left(10^{4}-10^{6}\right.$ years $)$ is heavily dependent on the ability to accurately predict its behavior with respect to potential release of radionuclides into the environment. Inevitably, such long-term predictions are available only from modeling techniques combined with available, short-term experimental data. The present concepts of waste repositories are based on multi-barrier systems part of which are clays and cementitious materials for their durability and capability to suppress migration of radionuclides $[1,2]$. The existing data are still insufficient to indicate how to prepare such materials with optimal properties, and further research in this area will be necessary.

One of the key aspects in this pursuit is to have accurate picture of which transport mechanisms are relevant to a given situation and to have accurate values of these transport parameters for given radionuclides, shielding materials, and external/internal conditions. This is an extremely complex task. Indeed, while diffusion is designed to be the main transport mechanism (the relevant parameter being a diffusion coefficient) [3], other transport mechanisms could be taken into account. These usually include advection in possible fractions, adsorption to pore walls, or radioactive decay [4]. Their corresponding parameters, respectively, are: the velocity and dispersion in fractions, their widths, density, etc.; parameters determining adsorption isotherm or adsorption kinetics; and the decay constant. However, even atypical mechanisms like surface diffusion and osmosis might turn

* Corresponding author: igor.medved@,fsv.cvut.cz 


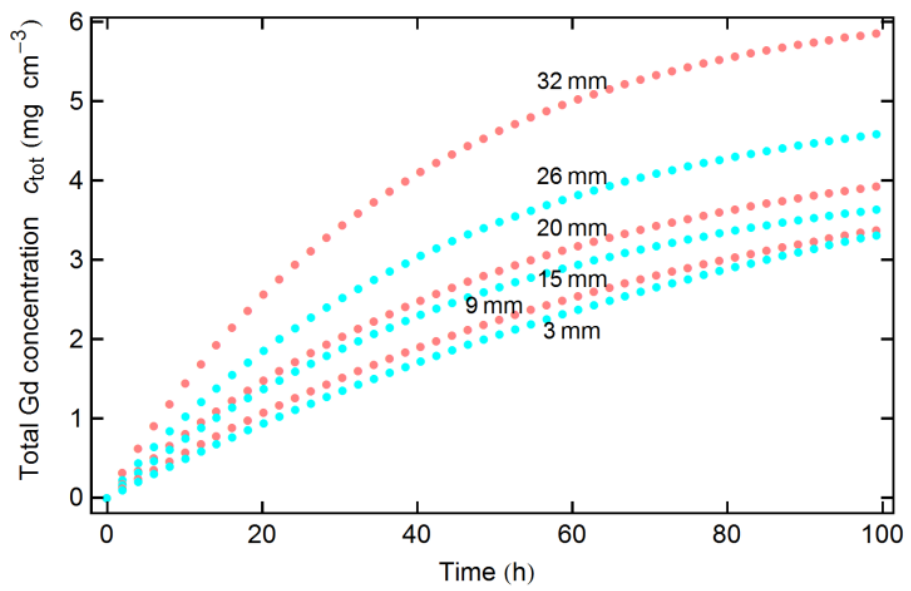

Fig. 1. The concentration profiles of gadolinium obtained from neutron radiography measurements. Each curve corresponds to a volume centered at the sample length as indicated, with its boundaries shifted plus and minus 3 millimeters from the center.

out to be present $[5,6]$. In addition, significant effects on radionuclides transport can be due to interactions with the shielding materials, for example, surface charge on pore walls may cause electrostatic exclusion [7], the $\mathrm{pH}$ of pore water may cause adsorption preference of cations/anions [8], and variations in the temperature may change the pore water viscosity and thus diffusivity [7]. Chemical effects (oxidation/reduction, precipitation and dissolution) could play a significant role too $[3,7]$.

In this paper we wish to present results and their analysis that concerns the use of neutron radiography [9] for the investigation of gadolinium transport in a cement composite. In comparison with other experimental techniques, neutron radiography has the great advantage of being simple in its application, non-destructive, and very precise. The so obtained data are studied by an inverse method in order to estimate the parameters (the diffusion coefficient, water diffusivity, and linear isotherm constant) of an advectiondiffusion model of Bear and Bachmat [10].

\section{Experimental}

The studied composite contained $29.1 \mathrm{wt} \%$ of cement CEM I $52.5 \mathrm{R}$ and $48.8 \mathrm{wt} \%$ of aggregate supplemented with silica flour and silica fumes $(6.6 \mathrm{wt} \%$ each). The aggregate was composed of four fractions of silica aggregate with grain sizes $1-4 \mathrm{~mm}(7.1 \mathrm{wt} \%), 0.6$ - $1.2 \mathrm{~mm}$ (9.7 wt\%), $0.3-0.8 \mathrm{~mm}(12.5 \mathrm{wt} \%)$, and $0.1-0.6 \mathrm{~mm}(19.5 \mathrm{wt} \%)$. The water/cement ratio was kept low ( $7.5 \mathrm{wt} \%$ of water) to maintain qualities of $\mathrm{HPC}$, and 1.3 $\mathrm{wt} \%$ of superplasticizer was added to maintain proper workability. The composite had the bulk density of $2160 \mathrm{~kg} \mathrm{~m}^{-3}$, the matrix density of $2395 \mathrm{~kg} \mathrm{~m}^{-3}$, and open porosity $9.8 \%$ [11]. The studied samples were cut from the prepared composite to have rectangular shape with cross section of $20 \mathrm{~mm} \times 20 \mathrm{~mm}$ and length of $55 \mathrm{~mm}$.

Neutron radiography was used to observe spatial and temporal concentration changes during the transport of gadolinium in the studied samples along their $55 \mathrm{~mm}$ side. We used solution of $\mathrm{GdNO}_{3}$ in which the concentration of $\mathrm{Gd}^{3+}$ was $200 \mathrm{mg} \mathrm{cm}{ }^{-3}$. The measurements were carried out on neutron diffractometer KSN-2 placed at the horizontal channel HK-2 of the research nuclear reactor LVR-15 located in Nuclear Research Institute in Rez near Prague, Czech Republic. The transport was characterized by registering 
temporal and spatial intensity changes in beam of monochromatic neutrons (wavelength of $0.1362 \mathrm{~nm}$, cross section of $15 \mathrm{~mm} \times 36 \mathrm{~mm}$ ) that penetrated through samples by neutron camera (Neutron Optics, France) and subsequent processing of the obtained 2D data by graphical program ImageJ. The data were measured in the time interval $0-500 \mathrm{~h}$. The resulting time and position dependence of the total gadolinium concentration in the studied sample is plotted in Fig. 1.

\section{Theoretical}

We model the transport of $\mathrm{Gd}^{3+}$ in the studied sample by a $1 \mathrm{D}$ version of the advectiondiffusion model of Bear and Bachmat [10]. The model includes dispersion in the liquid phase, influence of moisture flow on salt transport, and effect of bound salt on pore walls. Namely,

$$
\frac{\partial c_{\mathrm{tot}}}{\partial t}=\frac{\partial}{\partial x}\left(D w \frac{\partial c_{f}}{\partial x}+\kappa c_{f} \frac{\partial w}{\partial x}\right)
$$

where $c_{f}$ is the concentration of a free $\mathrm{Gd}$ in pore water and $c_{\text {tot }}=w c_{f}+c_{b}$ is the total concentration that includes both the free and bound Gd. The water content $w$ satisfies the mass balance equation

$$
\frac{\partial w}{\partial t}=\kappa \frac{\partial^{2} w}{\partial x^{2}}
$$

The diffusion coefficient $D$ of Gd and moisture diffusivity $\kappa$ are assumed to be constant here. In addition, we shall assume that a linear equilibrium sorption isotherm, $c_{b}=K_{d} c_{f}$, with a distribution coefficient $K_{d}$ can be applied.

The initial condition is chosen as $w(0, x)=c_{f}(0, x)=0$ (the sample was initially dry and devoid of Gd). The boundary conditions for the moisture content are $w(t, \infty)=0$ and $w(t, 0)$ $=w_{0}$, where the constant $w_{0}=0.098$ is chosen to coincide with the open porosity of the sample. The boundary conditions for the Gd concentration are chosen so that $c_{f}$ coincides with the data values at the smallest and largest positions $x_{1}$ and $x_{6}$.

When trying to fit the experimental data with the model from Eqs. (1) and (2), we applied the Levenberg-Marquardt method $[12,13]$. In fitting the data, this iterative procedure evaluates model parameters $\mathbf{p}$ by minimizing a positive least-square norm $S(\mathbf{p})$. In our case the parameters vector $\mathbf{p}$ is chosen as $\left(D, \kappa, K_{d}\right)$. The minimization is terminated when the parameters values $\mathbf{p}_{i+1}$ and $\mathbf{p}_{i}$ in the iteration step $i$ and $i+1$, respectively, are such that the conditions

$$
\sqrt{\frac{S\left(\mathbf{p}_{i+1}\right)}{N}} \leq \varepsilon_{1}, \quad \frac{\left\|\mathbf{p}_{i+1}-\mathbf{p}_{i}\right\|}{\left\|\mathbf{p}_{i+1}\right\|} \leq \varepsilon_{2}, \quad \frac{\left|S\left(\mathbf{p}_{i+1}\right)-S\left(\mathbf{p}_{i}\right)\right|}{S\left(\mathbf{p}_{i+1}\right)} \leq \varepsilon_{3} .
$$

are satisfied for prefixed values $\varepsilon_{1}, \varepsilon_{2}$, and $\varepsilon_{3}$ (here $N$ is the number of the data points). We set $\varepsilon_{2}=0.01$ and $\varepsilon_{3}=0.1$, while several values of $\varepsilon_{1}$ between 0.1 and 1 will be considered.

\section{Results and discussion}

When applying the Levenberg-Marquardt method to fit the experimental data with the 


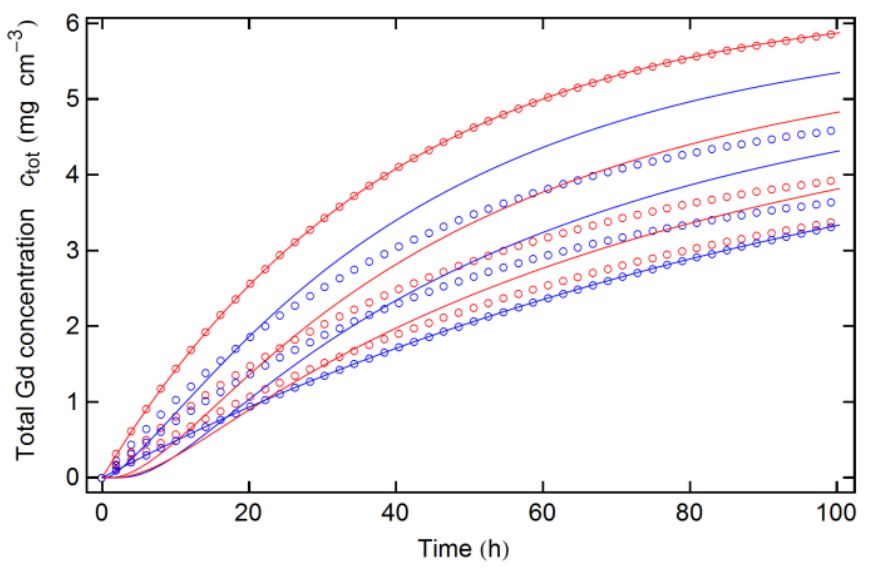

Fig. 2. The experimental data from Fig. 1 (circles) fitted with theoretical profiles (curves) obtained for the model parameters calculated with the Levenberg-Marquardt method.
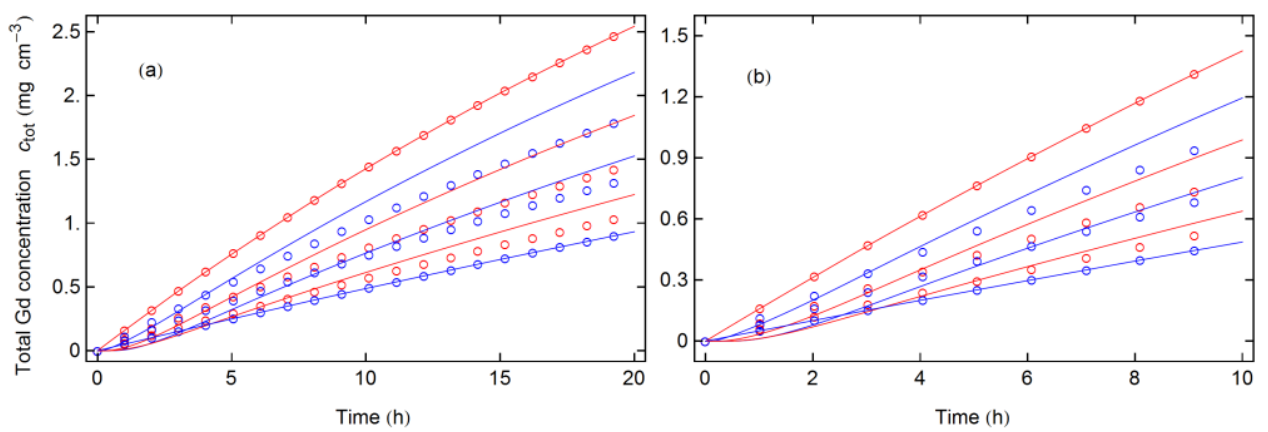

Fig. 3. The experimental data from Fig. 1 (circles) up to time (a) $20 \mathrm{~h}$ and (b) $10 \mathrm{~h}$ fitted with theoretical profiles (curves) obtained for the model parameters calculated with the LevenbergMarquardt method.

model from Eqs. (1) and (2), it turns out that the iterative procedure converges only when the three values $\varepsilon_{i}$ are chosen large enough: $\varepsilon_{1}=0.44, \varepsilon_{2}=0.0029$ and $\varepsilon_{3}=0.038$. The obtained values of the model parameters are $D=7.1 \times 10^{-8} \mathrm{~m}^{2} \mathrm{~s}^{-1}, \kappa=4.6 \times 10^{-8} \mathrm{~m}^{2} \mathrm{~s}^{-1}$, and $K_{d}=1.55$. A comparison of the total concentration calculated from Eqs. (1) and (2) for these parameters with experimental values are shown in Fig. 2. The agreement is only qualitative. The averaged relative discrepancy between the calculated and measured values of the total concentration is $19.1 \%$, the maximal being as large as $100 \%$.

To improve the precision, we also applied the Levenberg-Marquardt method to a smaller set of data points, namely, those occurring up to a time $t_{0}$. For $t_{0}=20 \mathrm{~h}$ the best precisions are $\varepsilon_{1}=0.15, \varepsilon_{2}=0.0055$ and $\varepsilon_{3}=0.048$ and the model parameters are $D=4.2 \times$ $10^{-7} \mathrm{~m}^{2} \mathrm{~s}^{-1}, \kappa=2.0 \times 10^{-7} \mathrm{~m}^{2} \mathrm{~s}^{-1}$, and $K_{d}=1.85$. Similarly, for an even shorter period, $t_{0}=10$ $\mathrm{h}$, the best precisions are $\varepsilon_{1}=0.07, \varepsilon_{2}=0.0032$ and $\varepsilon_{3}=0.039$ and the model parameters are $D=2.5 \times 10^{-7} \mathrm{~m}^{2} \mathrm{~s}^{-1}, \kappa=1.5 \times 10^{-7} \mathrm{~m}^{2} \mathrm{~s}^{-1}$, and $K_{d}=0.75$. Plots of the theoretical and experimental total concentrations for these two cases are shown in Fig. 3. Again, the agreement is only qualitative, although better than in Fig. 2 . For $t_{0}=20 \mathrm{~h}$ the averaged and maximal relative discrepancy between the calculated and measured values of the total concentration are $18.0 \%$ and $91 \%$, respectively; for $t_{0}=10 \mathrm{~h}$ they are $18.9 \%$ and $85 \%$, respectively. 
The above results indicate that the description of experimental results is more complex than it is provided by model from Eqs. (1) and (2). One drawback is the assumption of constant diffusion coefficients $D$ and $\kappa$. A more general and appropriate approach would be to consider diffusion coefficients $D$ and $\kappa$ that vary with $c_{f}$ and $w$, respectively. A more complicated (equilibrium or kinetic) mechanism of adsorption of Gd may be also taken into account. Finally, non-diffusion mechanisms of Gd transport could be necessary to be included, such as surface diffusion [5] or osmosis [6]. To be able to analyze the transport of Gd more precisely, it will be necessary to perform further experiments - both for water saturated samples and for dry samples with pure water.

\section{Conclusions}

We investigated the transport of gadolinium in a dry cement composite using neutron radiography and an inverse approach using an advection-diffusion model with linear adsorption. Neutron radiography turns out to be a very efficient tool in experimental studies of transport phenomena, providing rather accurate concentration profiles of the transported species. The physical interpretation of the data with constant model parameters was successful only qualitatively, while quite large quantitative discrepancies can occur between the data and calculated results. We suggested that further experimental and theoretical research would be necessary to better understand not only the studied transport process but also other analogous transports of nuclides in similar porous materials.

This research was supported by the Czech Science Foundation under the project No. 17-11635S and by the Slovak Research and Development Agency under the project No. APVV-15-0681. The support of the neutron radiography method from the OPRDE MEYS CR project CZ.02.1.01/0.0/0.0/16_019/0000778 is also acknowledged.

\section{References}

1. B. Ma, L. Charlet, A. Fernandez-Martinez, M. Kang, B. Madé, Appl. Geochem. 100, 414 (2019)

2. J. Kot’átková, J. Zatloukal, P. Reiterman, K. Kolář, J. Environ. Radioactiv. 178-179, 147 (2017)

3. C. D. Shackelford, S. M. Moore, Eng. Geol. 152, 133 (2013)

4. D. H. Tang, E. O. Frind, E. A. Sudicky, Water Resour. Res. 17, 555 (1981).

5. I. Medved', R. Černý, Micropor. Mesopor. Mater. 142, 405 (2011)

6. I. Medved', R. Černý, Micropor. Mesopor. Mater. 170, 299 (2013)

7. N. G. Sawatsky, D. W. Oscarson, Soil Sci. Soc. Am. J. 55, 1261 (1991)

8. H. L. Bohn, B. L. McNeal, G. A. O'Connor, Soil Chemistry (2nd ed., John Wiley and Sons Inc., New York, 1985)

9. I. S. Anderson, R. L. McGreevy, H. Z. Bilheux, Neutron Imaging and Applications: A Reference for the Imaging Community (Springer, New York, 2009)

10. J. Bear, Y. Bachmat, Introduction to modelling of transport phenomena in porous media (vol. 4, Kluwer, Dordrecht, 1990)

11. M. Čáchová, J. Kot’átková, M. Doleželová, E. Vejmelková, P. Konvalinka, R. Černý, Key Eng. Mat. 760, 127 (2018)

12. K. Levenberg, Quart. Appl. Math. 2, 164 (1944)

13. D. W. Marquardt, J. Soc. Ind. Appl. Math. 11, 431 (1963) 